ness of food in times of plenty; and make the dearness of food in times of scarcity appear far less serious than it really is.

One of the most important practical results of this investigation probably is, that it affords a certain amount of power to predict the variations of prices in the coming sunspot cycle. Of course, until all those fluctuations which appear at present to be subject to no law have been explained and reduced to order, if ever that should be possible, exact prediction in any individual case is altogether out of the question, but as there is a regularly recurring eleven-yearly wave of prices running through the irregular fluctuations and following the sunspot wave in the manner defined by the curves, it is possible to form an estimate of the general level of prices in the different years of the coming sunspot cycle. There is thus some reason for believing that the present period of low prices following the last maximum of sunspots, which appears to have occurred about the end of 1882 or early in 1883 , will not last very much longer, a brisk rise of prices being due in the Deccan and in Madras five years after the sunspot maxinum, that is, in 1887 or I 888 , and in more northern districts a year or two later.

This estimate will, of course, be subject to modification if it should be found that the sunspot curve is declining towards its minimum more or less rapidly than usual. The last period of sunspots appears to have been somewhat longer than the average, that is, about twelve years from the maximum of 1870 to that of 1882 , instead of the normal length of almost exactly eleven years; and the coming minimum may possibly follow the last maximum more quickly than usual. Fortunately, the sunspot observations are not the only indicators of this cosmical periodicity, for, as I have shown in a paper communicated to the Royal Society in 1884 , the magnetic observations recorded at the Colaba Observatory afford far smoother and more definite indications of this periodicity than the sunspot observations ; and, what is even more important, the eleven-yearly magnetic variation precedes the sunspot variation by almost exactly six months, so that the magnetic indications are given half a year earlier than those of the sunspots.

Bombay, April 1886

\section{THE PHYSICAL APPEARANCE OF MARS} $I N \mathbf{I} 886$

A SERIES of observations of Mars were obtained here in March and April last with a ro-inch silveron-glass reflector by With of Hereford. The powers employed were 252 and 475 , but I found no advantage from the latter, which seemed too high for the purpose. As a rule a single lens magnifying 252 was amply sufficient, though there were several occasions when a power of about 350 would have been a decided acquisition.

The planet came to opposition on March 6, but during the first three weeks of March we had intense frosts, and it was not feasible to commence observations until towards the end of that month. The opposition magnitude of Mars was only $16^{\prime \prime} \cdot 6$, so that as regards apparent diameter the planet was far from being favourably placed. At the opposition of 1877 the diameter was no less than $29^{\prime \prime} 5$. But at the recent opposition the north hemisphere of the planet (which has not hitherto been so thoroughly examined as the south hemisphere, and does not exhibit so many striking features) was well presented for observation, the latitude of the centre of the disk being about $22^{\circ} \mathrm{N}$. in March and April.

The markings seen were both numerous and diversified. There is evidently a mass of detail on the planet, which is, however, most difficult to trace out in reliable characters. Many faint lineaments reach the eye with sufficient distinctness to prove their existence, but they cannot be held steadily enough or with that perspicuity to allow of the delineation of their outlines, or to enable their relative positions to be correctly assigned. Only the more pronounced features can be drawn satisfactorily. The small diameter of Mars during the recent observations has in a great measure induced this uncertainty as to the physical aspect of the disk. Another cause is found in the rarity of really good telescopic images. Not only must the atmosphere be peculiarly favourable to sharp definition, but there must be an absence of wind. A complicated system of markings cannot be made out under the influence of annoying vibrations. Moreover, this planet, considered as a telescopic object, is far less satisfactory than either Jupiter or Saturn, and this circumstance, with the other drawbacks alluded to, have given rise to that uncertainty, and to many of the discordances, in regard to the visible markings observed on his surface.

My intention in the present paper is merely to describe general results, as a particular description would scarcely be intelligible without drawings. Between March 23 and April 30 the planet was examined on twenty-one evenings, and a considerable number of sketches were completed. During the period mentioned the weather afforded an unusual number of clear nights, and whenever the seeing was fairly good the visible features were carefully noted, the results being afterwards compared with each other and with former work in the same direction. My drawings correspond very closely amongst themselves, and there is a fair agreement in the main features with those depicted on the charts of Green, Schiaparelli, Knobel, and others. I have also compared them with the views given in Terby's work on Mars and with Boeddicker's drawings of I88I and I 884 (with Lord Rosse's 3-foot reflector) published in the scientific Transactions of the Royal Dublin Society, and find in many instances a substantial confirmation. Some of the differences are larger than would have been considered probable, but experience has taught us that it is useless to expect uniformity in delineations of planetary details.

During the five weeks over which my observations extended I saw no conclusive evidences of physical changes in any of the markings. But the period was too limited, and the circumstances affecting the review altogether too unfavourable, to enable me to speak definitely on this point. The slight differences apparent amongst my drawings are merely such as were occasioned by changes in local atmospheric conditions. On a bad night faint markings, previously distinguished, would appear obliterated, and on thoroughly good nights I saw delicate appearances which were utterly beyond reach on less auspicious occasions. I am convinced that these changes in the character of the seeing, exercise great influence on the distinguishable features of a planet ; more so, in fact, than observers usually concede. Inferences of real change are sometimes hastily adopted in consequence, but they can only be substantiated after the most searching examination and the most convincing proofs.

The exterior edges of many of the well-defined seas on Mars are very brilliant, and their boundaries very definite. These brilliant outlying borders remind one of the light areas often abutting on the dark spots of Jupiter, only in the case of Mars they are more extensive, more permanent, and altogether dissimilar in form. I may instance a particular case of this bright bordering in the immediate region east of the Kaiser Sea on Mars. On several occasions this was so striking as to vie with the bright patch about the north pole. This shimmering extends several degrees east of the dark outline of the sea, but is limited by a faint and irregularly-condensed marking extending northwards, with an inclination east, from the knot in longitude $290^{\circ}$ just east of the north extremity of the Kaiser Sea as figured in Mr. Green's chart. This marking runs over a considerable tract, and its east extension underlies Davies' forked bay and Burton Bay, to both of which it is connected by faint ligaments of shade, 
reminding one of the "canals" of Schiaparelli. This special marking, which is not included in Mr. Green's map, may be identical with the network of dark narrow streaks figured in this region by Schiaparelli in his chart for January and February, I882. It is also more or less definitely shown in some other drawings, notably on one by Schmidt, which forms No. I7 in Dr. Terby's Areography.

As to the Kaiser Sea, it appears very faint and narrow, if not really broken, in the region some $10^{\circ}$ or $15^{\circ}$ south of its north extremity. This peculiarity is well drawn in Herr Boeddicker's drawings of December 27, November 19, and December 26, 1881 (Nos. I1, I3, and 14) in the scientific Transactions of the Royal Dublin Society for December, I 882 . Consulting other drawings I cannot find that this feature is sufficiently indicated. It is obvious, however, that it would only be well detected when placed near the apparent centre of the disk as during the recent opposition.

Mr. Knobel's drawings in 1873 (Monthly Notices, vol. xxxiii., facing p. 476) agree generally with mine far closer than those he has published in the Memoirs, vol. xlviii. part ii., I 884. I always see Knobel Sea on Green's chart separated on its south side from the fainter curving band running east, as in the sketches Nos. 6, 7, 8, and 9, 1873 . This break is not depicted in the subsequent drawings of I884, so that the appearance has either been subject to actual variation of aspect or the difference of inclination has originated the want of uniformity. Probably the latter is the real cause, for the inclination of Mars in April and May, 1873, was nearly identical with that of March and April, I886, and it is for these periods the drawings are so nearly alike in their more conspicuous forms. I see the northern boundary of Knobel Sea distinctly separate from the dark longitudinal strip immediately contiguous to the north polar cap. The drawing No. I2, May 19, I873, by Mr. Knobel, portrays the leading features of this region much as I have more recently observed them. In $\mathrm{I} 884, \mathrm{Mr}$. Knobel delineated the whole mass of shading outlying the north pole as blended uninterruptedly, but these differences are unquestionably due to the changes of inclination, which must necessarily introduce such ciiscordances into the apparent forms of the markings as observed at different epochs.

As to the canal-shaped features of Schiaparelli, first seen in 1877 and 1878 , and subsequently confirmed, I have distinguished a large number of appearances highly suggestive of such a configuration, but the Italian drawings made during the three months from. October, I881, to February, I882, give them a definite character, and (apart from their duplication) a straightness of direction and general uniformity of tone which my observations do not confirm. The more delicate and complex markings on the planet appear to my eye, under the best circumstances, as extremely faint, linear shadings with evident gradations in tone and irregularities occasioning breaks and condensations here and there. If they existed under the same aspect and with the same boldness as delineated by Schiaparelli, they would have been readily detected here whenever the seeing was fairly good, for these objects are referred to as readily observed in the 8-inch refractor of the Milan Observatory in February, 1882 , when the planet's diameter was only $\mathrm{I}^{\prime \prime}$. The duplication of these lines was also traceable under the same unfavourable conditions. The wonder is, not that the eminent Italian astronomer has discovered such a marvellous extent of curious detail on this planet, because this detail unquestionably exists, though scarcely in the form and character under which it is represented, but that he should have observed its more complex and difficult configuration at the very period when Mars was so very unfavourably situated for observations of this critical nature.

The surface markings of this planet are so numerous and varied that they are far from being adequately represented on existing charts. In certain regions the disk is so variegated as to give a mottled appearance. Dark lines, and spots, and bright spaces are so thickly interspersed, and so difficult to observe with sufficient steadiness to estimate their positions and forms, that I found it impossible to make thoroughly satisfactory drawings. An observer has to be content with endeavours to depict the more prominent marks only, and even in connection with these there is always some element of uncertainty. The rotation-period of the planet is, however, so slow, the hourly rate being only $14^{\circ} .6$ in comparison with $36^{\circ} 7$ in the case of Jupiter, that plenty of time is afforded for drawing the leading markings before they show a displacement obvious to the eye. In addition to this a drawing of Mars may be made to rest on several successive evenings of observation if the observer comes $37 \%$ min. later to the telescope on each occasion. In regard to Jupiter the difficulty of suitably drawing the details is far greater, though they admit of more ready observation. The rapid rotation of this planet displaces objects in a few minutes, and makes it imperative that the work both of observing and charting should be very hastily performed; and it is not feasible in this case to base a sketch on observations of following nights, because the markings are influenced by different velocities, and suffer large relative displacements even at short intervals of time.

During the past few months the north polar cap of Mars has been very bright, sometimes offering a startling contrast to those regions of the surface more feebly reflective. Some of the other parts were also notably brilliant. These luminous regions of Mars require at least as much careful investigation as the darker parts, for it is probably in connection with them that physical changes (if at present operating on the planet's surface) may be definitely observed. In many previous drawings and descriptions of Mars sufficient weight has not been accorded to these white spots.

Many of our leading treatises on astronomy attribute a dense atmosphere to Mars, but nothing has been observed during my recent observations to corroborate this theory. It seems to me far more plausible to assume that the atmosphere of this planet is extremely attenuated. The chief spots are invariably visible, and the phenomena occasionally observed are rather to be imputed to the vagaries of our own atmosphere than to that of Mars. Jupiter and Saturn are doubtless enveloped in dense vapours shrouding their real surfaces from terrestrial eyes. Their markings are atmospheric, though in some cases very durable, and constantly undergoing changes of aspect and displacements of position by longitudinal currents. On Mars a totally different nature of things prevails. Here the appearances descried are absolute surface markings displaying none of the variations which are so conspicuously displayed in the markings on Jupiter. It is probable that many, if not all, the changes supposed to have occurred in the features of Mars are simply attributable to the constantly varying conditions under which the planet has necessarily to be observed. Were the circumstances of observation more equable there would be much greater unanimity amongst observers of this interesting object. It seems to me that the very pronounced character of the markings and their great permanency are quite opposed to the idea that the planet is surrounded by a dense cloud-laden atmosphere.

W. F. DENNING

\section{CORNU ON THE HYDROGEN FUNCTION} OF CERTAIN METALS ${ }^{1}$

\THEN we examine on different photographs those groups of lines which reappear periodically with a particular regularity, we find that these groups belong $x$ Translation from an article in the Joumal de Physigue. 